\title{
$\hbar$-independent universality of the quantum and classical canonical transformations
}

\author{
T. Hakioğlu ${ }^{\mathrm{a}, *}$, A. Teğmen ${ }^{\mathrm{b}}$, B. Demircioğlu ${ }^{\mathrm{c}}$ \\ a Physics Department, Bilkent University, 06533 Ankara, Turkey \\ b Physics Department, Ankara University, 06100 Tandoğan, Ankara, Turkey \\ c Sarayköy Nuclear Research and Training Center, 06983 Kazan, Ankara, Turkey
}

Received 24 May 2006; received in revised form 22 August 2006; accepted 24 August 2006

Available online 1 September 2006

Communicated by P.R. Holland

\begin{abstract}
A theory of non-unitary-invertible and also unitary canonical transformations is formulated in the context of Weyl's phase space representations. It is shown in the phase space that all quantum canonical transformations without an explicit $\hbar$ dependence are also classical mechanical and vice versa. Contrary to some earlier results, it is also shown that the quantum generators and their classical counterparts are identical in this $\hbar$-independent universal class.

(c) 2006 Elsevier B.V. All rights reserved.
\end{abstract}

PACS: 03.65.-w; 02.30.Uu; 04.60.Ds

\section{Introduction}

Canonical transformations (CTs) played a crucial role in the historical development of quantum mechanics $[1,2]$. So profound the contribution of the transformation theory to the fundamental understanding of quantum mechanics is that it is just to compare it [3] to the beginning of a new phase in analytical dynamics initiated by Poisson in the generalized coordinates and later by Jacobi, Poincaré, Appell and Hamilton in the development of the canonical formalism. While the development in the early phases of quantum mechanics was characterized by the configuration and phase space approaches, its later elaborations led to the conception of abstract Hilbert space through which the formerly important transformation theory approach lost its momentum [3]. Contrary to the case with the wellformulated linear CTs [4], formulating the non-linear ones is made more challenging in the presence of deep problems as in-

\footnotetext{
* Corresponding author.

E-mail addresses: hakioglu@ @en.bilkent.edu.tr (T. Hakioğlu), tegmen@science.ankara.edu.tr (A. Teğmen), bengu@taek.gov.tr (B. Demircioğlu).
}

vertibility, uniqueness [1], unitarity versus non-unitarity [1,5], and, in many cases, even the lack of the transformation generators in connection with the absence of the identity limit [7]. They mediate a unique language with the path integral quantization at one extreme $[8,9]$ and the Fresnel's geometrical optics on the other [10]. Their unitary representations were first treated by Dirac [2] as a first step towards the path integral quantization.

In 1927 Weyl [11] introduced a new quantization scheme based on a generalized operator Fourier correspondence between an operator $\hat{\mathcal{F}}=\mathcal{F}(\hat{p}, \hat{q})$ and a phase space function $f(p, q)$. To observe the Dirac correspondence as a special case, Weyl restricted the space of the operator to the Hilbert-Schmidt space where monomials such as $\hat{p}^{m} \hat{q}^{n}$ acquire finite norm for all $0 \leqslant m, n$. Weyl's formalism was then extended by the independent works of von Neumann, Wigner, Groenewold and Moyal [12] to a general phase space correspondence principle between the operator formulation of quantum mechanics and its equivalent version on the non-commutative phase space.

There has been some reviving interest in the quantum CTs and their classical limits [7,13-16]. The goal of this paper is to formulate the quantum CTs within phase space covariant 
formulation of Weyl quantization. More importantly, it is also shown that the Weyl quantization allows (contrary to some conventional belief, see Ref. [6]) a restricted covariance under certain types of non-linear CTs.

\section{Weyl quantization and canonical transforms}

According to the Weyl scheme a Hilbert-Schmidt operator $\hat{\mathcal{F}}$ is mapped one-to-one and onto to a phase space function $f(p, q)$ as

$$
\begin{aligned}
& f(p, q)=\operatorname{Tr}\{\hat{\Delta}(p, q) \hat{\mathcal{F}}\}, \\
& \hat{\mathcal{F}}=\int \frac{1}{\hbar} \frac{d p}{2 \pi} \frac{d q}{2 \pi} f(p, q) \hat{\Delta}(p, q), \\
& -\infty<p, q<\infty,
\end{aligned}
$$

where

$$
\begin{aligned}
& \hat{\Delta}(p, q)=\int d \alpha d \beta e^{-i(\alpha p+\beta q) / \hbar} e^{i(\alpha \hat{p}+\beta \hat{q}) / \hbar}, \\
& -\infty<\alpha, \beta<\infty
\end{aligned}
$$

is an operator basis satisfying all the necessary conditions of completeness and orthogonality of the generalized Fourier operator expansion. The phase space function $f(p, q)$ is often referred to as the phase space symbol of $\hat{\mathcal{F}}$. The operator product corresponds to the non-commutative, associative $\star$ product $\hat{\mathcal{F}} \hat{\mathcal{G}} \Longleftrightarrow f \star g$,

$\hat{\mathcal{F}} \hat{\mathcal{G}} \hat{\mathcal{H}} \Longleftrightarrow f \star g \star h$,

where $\hat{\mathcal{F}}, \hat{\mathcal{G}}, \hat{\mathcal{H}}$ and their respective symbols $f, g, h$ are defined by (1) and (2). The $\star$-product is a formal exponentiation of the Poisson bracket $\stackrel{\leftrightarrow}{\mathcal{D}}_{(q, p)}$ as

$\star_{(q, p)} \equiv \exp \left\{\frac{i \hbar}{2} \stackrel{\leftrightarrow}{\mathcal{D}}_{(q, p)}\right\}=\sum_{n=0}^{\infty}\left(\frac{i \hbar}{2}\right)^{n} \frac{1}{n !}\left[\stackrel{\leftrightarrow}{\mathcal{D}}_{(q, p)}\right]^{n}$

$\stackrel{\leftrightarrow}{\mathcal{D}}_{(q, p)}=\frac{\overleftarrow{\partial}}{\partial q} \frac{\vec{\partial}}{\partial p}-\frac{\overleftarrow{\partial}}{\partial p} \frac{\vec{\partial}}{\partial q}$

where the arrows indicate the direction that the partial derivatives act. Unless specified by arrows as in (4), their action is implied to be on the functions on their right. According to (3) the symbol of the commutator is defined by the Moyal bracket

$$
[\hat{\mathcal{F}}, \hat{\mathcal{G}}] \quad \Leftrightarrow \quad\{f(p, q), g(p, q)\}_{q, p}^{(M)}=f \star_{q, p} g-g \star_{q, p} f
$$

which has a crucial role in deformation quantization [17]. In the latter, the Moyal bracket is a representation of the quantum commutator in terms of a non-linear partial differential operator, and at the same time it is an $\hbar$-deformation of the classical Poisson bracket. The canonical commutation relation (CCR) between the canonical operators, say $\hat{P}, \hat{Q}$, is represented by the phase space symbols of these operators denoted respectively by $P(p, q), Q(p, q)$. If $[\hat{P}, \hat{Q}]=-i \hbar$ then

$$
\begin{aligned}
\{P & Q\}_{q, p}^{(M)} \\
& =2 \sum_{k=0}^{\infty}\left(\frac{i \hbar}{2}\right)^{2 k+1} \frac{1}{(2 k+1) !} P(p, q)\left[\stackrel{\leftrightarrow}{\mathcal{D}}_{(q, p)}\right]^{2 k+1} Q(p, q) \\
& =-i \hbar
\end{aligned}
$$

It is well known that, a large class of CT can be represented by not only unitary but also non-unitary (and invertible) operators [1] whose action preserve the CCR. Counter examples to unitary transformations [5] are abound and some of the distinct ones are connected with the multi-valued (non-invertible) or domain non-preserving (non-unitary and invertible) operators. A few examples can be given by the polar-phase-space [5] (i.e. action-angle) and quantum Liouville transformation [18] which are multi-valued transformations, or Darboux type transformations between iso-spectral Hamiltonians [7].

Here we will reformulate the quantum canonical (unitary as well as non-unitary) transformations within the Weyl formalism paying specific attention to a particular subclass of them characterized by no explicit $\hbar$ dependence in the canonical variables $P(p, q)$ and $Q(p, q)$. The importance of this particular class is that, thinking of $\hbar$ as a free parameter, the only non-zero contribution to the $\hbar$ expansion of the canonical Moyal bracket in (5) is the first (i.e. $k=0$ ) term

$$
\{P, Q\}_{q, p}^{(M)}=i \hbar\{P, Q\}_{q, p}^{(P)}+\left.\mathcal{O}\left(\hbar^{2 k+1}\right)\right|_{1 \leqslant k} \mapsto-i \hbar
$$

yielding

$$
\{P, Q\}_{q, p}^{(M)}=i \hbar\{P, Q\}_{q, p}^{(P)},
$$

where all $\mathcal{O}\left(\hbar^{2 k+1}\right)$ terms with $1 \leqslant k$ necessarily vanish. (In Eqs. (6) and (7) the superscript $P$ stands for the Poisson bracket.) Eq. (7) is the statement that the classical and quantum canonical $\hbar$-independent transformations are identical in the group theory sense yielding the strong result that their generating functions should also be identical. From the Lie algebraic perspective, the equivalence of the classical and quantum generators has been established in Ref. [15]. This proof obviously contradicts with some earlier results $[6,16]$ in which the Moyal covariance stated in (7) was overlooked.

We also observe that (7) holds between the canonical pairs, whereas it is not generally true for arbitrary functions $f(p, q)$ and $g(p, q)$. Eq. (7) states an equivalence between the canonical Moyal and the canonical Poisson brackets for $\hbar$ independent transformations.

The result in (7) implies that an $\hbar$ independent quantum CT is also a classical CT, a result that was obtained by Jordan [1] long time ago using a semiclassical approach.

\section{The phase space images of canonical transformations}

The Weyl formalism is restricted to a subspace of the Hilbert space in which the state functions decay sufficiently strongly at the boundaries to admit an infinite set of finite valued phase space moments $\hat{p}^{m} \hat{q}^{n}$ with non-negative integers $m, n$. If the moments are symmetrically ordered (i.e. Weyl ordering) we denote them by $\hat{t}_{m, n}=\left\{\hat{p}^{m} \hat{q}^{n}\right\}$. The $\hat{t}_{m, n}^{(0)}$ 's are simpler to represent in the phase space and they correspond to the monomials $p^{m} q^{n}$. A function $f(p, q)$ which can be written as a double Taylor expansion in terms of the monomials $p^{m} q^{n}$ corresponds to a symmetrically ordered expansion of an operator $\hat{\mathcal{F}}$ as

$$
f(p, q)=\sum_{0 \leqslant(m, n)} f_{m, n} p^{m} q^{n} \Leftrightarrow \hat{\mathcal{F}}=\sum_{0 \leqslant(m, n)} f_{m, n} \hat{t}_{m, n}^{(0)} .
$$


Symmetrically ordered monomials are Hermitian and they can be convenient in the expansion of other Hermitian operators.

The phase space representations are more convenient to use than the operator algebra for keeping track of $\hbar$ 's. Since $\hat{t}_{m, n} \Leftrightarrow$ $p^{m} q^{n}$, intrinsic $\hbar$ dependencies appear only in the phase space expansions representing non-symmetrical monomials. Suppose that the operator $\hat{\mathcal{F}}$, which has the Weyl representation $f(p, q)$, is transformed by an operator $\hat{U}$ which has the Weyl representation $u(p, q)$ by $\hat{\mathcal{F}}^{\prime}=\hat{U}^{-1} \hat{\mathcal{F}} \hat{U}$. Assume that the transformation $\hat{U}$ is given in an exponential form $\hat{U}_{\mathcal{A}}=e^{i \gamma \hat{\mathcal{A}} / \hbar}$ where $\gamma$ is a continuous parameter and the generator $\hat{\mathcal{A}}=\mathcal{A}(\hat{p}, \hat{q})$ is expanded ala (8) as

$\mathcal{A}(\hat{p}, \hat{q})=\sum_{m, n} a_{m, n} \hat{t}_{m n}^{(0)}$,

where $a_{m, n}$ 's are the expansion coefficients. We then have by $\hat{\mathcal{F}}^{\prime}=\hat{U}^{-1} \hat{\mathcal{F}} \hat{U}$ and Eq. (1)

$f^{\prime}(p, q)=\operatorname{Tr}\left\{\hat{\mathcal{F}}^{\prime} \hat{\Delta}\right\}=\operatorname{Tr}\left\{\hat{\mathcal{F}} \hat{U}_{\mathcal{A}} \hat{\Delta} \hat{U}_{\mathcal{A}}^{-1}\right\}$,

$\hat{U}_{\mathcal{A}} \hat{\Delta} \hat{U}_{\mathcal{A}}^{-1}=\hat{\Delta}+\frac{i \gamma}{\hbar}[\hat{\mathcal{A}}, \hat{\Delta}]+\frac{(i \gamma)^{2}}{2 ! \hbar^{2}}[\hat{\mathcal{A}},[\hat{\mathcal{A}}, \hat{\Delta}]]+\cdots$.

The right-hand side of (10) can be represented by certain linear first order phase space differential operators producing the left and right action of $\hat{p}$ and $\hat{q}$ on $\hat{\Delta}$ as [19]

$$
\begin{aligned}
& \hat{p} \hat{\Delta}(p, q)=\underbrace{\left[p+\frac{i \hbar}{2} \frac{\partial}{\partial q}\right]}_{\hat{p}_{L}} \hat{\Delta}(p, q), \\
& \hat{\Delta}(p, q) \hat{p}=\underbrace{\left[p-\frac{i \hbar}{2} \frac{\partial}{\partial q}\right]}_{\hat{p}_{R}} \hat{\Delta}(p, q), \\
& \hat{q} \hat{\Delta}(p, q)=\underbrace{\left[q-\frac{i \hbar}{\partial p} \frac{\partial}{\partial \hbar}\right]}_{\hat{q}_{L}} \hat{\Delta}(p, q), \\
& \hat{\Delta}(p, q) \hat{q}=\underbrace{\left[q+\frac{i \hbar}{2} \frac{\partial}{\partial p}\right]}_{\hat{q}_{R}} \hat{\Delta}(p, q)
\end{aligned}
$$

and thus,

$$
\begin{aligned}
{\left[\hat{t}_{m, n}, \hat{\Delta}(p, q)\right] } & =\left\{\hat{p}_{L}^{m} \hat{q}_{L}^{n}-\hat{p}_{R}^{m} \hat{q}_{R}^{n}\right\} \hat{\Delta}(p, q) \\
& \equiv \hat{S}_{m, n} \hat{\Delta}(p, q),
\end{aligned}
$$

where we used the specific notation $\hat{S}_{m, n}$ for the image of the symmetric monomials $\hat{t}_{m, n}$. Using Eqs. (11), the first commutator in the expansion in (10) becomes

$[\hat{\mathcal{A}}, \hat{\Delta}]=\hat{V}_{\mathcal{A}} \hat{\Delta}(p, q)$,

where $\hat{V}_{\mathcal{A}}$ is the Moyal-Lie representation [15] of the generator $\mathcal{A}$ given by

$\hat{V}_{\mathcal{A}}=\sum_{m, n} a_{m, n}\left\{\hat{p}_{L}^{m} \hat{q}_{L}^{n}-\hat{p}_{R}^{m} \hat{q}_{R}^{n}\right\}$
The right-hand side of (10) can be obtained by infinitely iterating the commutator (13) which yields

$\hat{U}_{\mathcal{A}} \hat{\Delta} \hat{U}_{\mathcal{A}}^{-1}=e^{i \gamma \hat{V}_{\mathcal{A}} / \hbar} \hat{\Delta}$.

Using Eq. (15) in (10)

$f^{\prime}(p, q)=e^{i \gamma \hat{V}_{\mathcal{A}} / \hbar} f(p, q)$.

There exists a linear map, for given $\hat{\mathcal{A}}$, such that $[, \hat{\Delta}]: \hat{\mathcal{A}} \mapsto$ $\hat{V}_{\mathcal{A}} \hat{\Delta}$. It is trivial that $\hat{\mathcal{C}}=\alpha \hat{\mathcal{A}}+\beta \hat{\mathcal{B}}$ is mapped as $\hat{V}_{\mathcal{C}}=\alpha \hat{V}_{\mathcal{A}}+$ $\beta \hat{V}_{\mathcal{B}}$. Thus $[\hat{\mathcal{A}}, \hat{\mathcal{B}}]$ is mapped as

$\hat{V}_{[\mathcal{A}, \mathcal{B}]}=-\left[\hat{V}_{\mathcal{A}}, \hat{V}_{\mathcal{B}}\right]$

via the Jacobi identity. Hence, if the closed set $\left\{\hat{\mathcal{A}}_{i}\right\}$ are generators of a Lie algebra then their images $\hat{V}_{\mathcal{A}_{i}}$ are generators of the Moyal-Lie algebra [15].

The Weyl correspondence including the covariance under canonical transformations can now be summarized in the commuting diagram

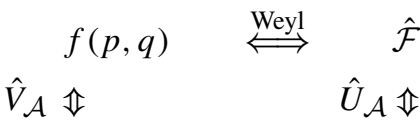

$$
\begin{aligned}
& f^{\prime}=e^{i \gamma \hat{V}_{\mathcal{A}} / \hbar} f \stackrel{\text { Weyl }}{\Longleftrightarrow} \quad \hat{\mathcal{F}}^{\prime} .
\end{aligned}
$$

The meaning of the diagram (18) can be facilitated by an example. Consider, for instance, the unitary transformation corresponding to $\hat{U}_{2,1}$. Using Eqs. (11) and (12) we find the corresponding differential generator $\hat{S}_{2,1}$ as

$\hat{V}_{\mathcal{A}}=\hat{S}_{2,1}=i \hbar\left(2 p q \partial_{q}-p^{2} \partial_{p}+\frac{\hbar^{2}}{4} \partial_{q}^{2} \partial_{p}\right)$

which has an explicit overall $\hbar$ dependence. Also note that $\hat{S}_{2,1}$ is an Hamiltonian vector field. For any $f(p, q)$ its action gives the Poisson (and Moyal) bracket

$$
\hat{S}_{2,1} f(p, q)=i \hbar\left\{f(p, q), p^{2} q\right\}^{(P)}=\left\{f(p, q), p^{2} q\right\}_{q, p}^{(M)} .
$$

Let us consider for $f$ and $f^{\prime}$ in the diagram (18) the canonical coordinates $(p, q)$ and $(P, Q)$. Then, using Eq. (19)

$P(p, q)=e^{-i \gamma \hat{S}_{2,1} / \hbar} p=\frac{p}{1+\gamma p}$,

$Q(p, q)=e^{-i \gamma \hat{S}_{2,1} / \hbar} q=q(1+\gamma p)^{2}$,

such that $P^{2} Q=p^{2} q$. It can be directly observed that the canonical transformation in Eq. (21) respects (7).

\section{Generating functions}

The Weyl symbol of an admissible operator $\hat{U}$ is given by,

$\hat{U}=\int \frac{d p d q}{(2 \pi)^{2} \hbar} u(p, q) \hat{\Delta}(p, q)$.

Since $\hat{U}$ is unitary, then $u(p, q)$ satisfies $u^{*}(p, q)=u^{(-1)}(p, q)$ where $*$ denotes the complex conjugation and the $u^{(-1)}$ is the Weyl symbol of $\hat{U}^{-1}$. Eq. (22) also converts an inner product in 
the Hilbert space to that in the phase space. The former is given by

$$
\begin{aligned}
(\psi, \hat{U} \varphi) & =\int d q \psi^{*}(q)(\hat{U} \varphi)(q) \\
& =\int \frac{d p d q}{(2 \pi)^{2} \hbar} u(p, q)(\psi, \hat{\Delta}(p, q) \varphi) .
\end{aligned}
$$

Using the matrix elements $\langle y|\hat{\Delta}(p, q)| x\rangle$ and considering a functional derivative of (23) with respect to $\psi^{*}(y)$, we find in the coordinate-coordinate representation that

$$
\begin{aligned}
& (\hat{U} \varphi)(y)=\int d x e^{i F(y, x)} \varphi(x), \\
& e^{i F(y, x)}=\int \frac{d p}{2 \pi \hbar} e^{-i p(x-y) / \hbar} u\left(p, \frac{x+y}{2}\right) .
\end{aligned}
$$

For the mixed (coordinate-momentum) representation

$$
\begin{aligned}
(\hat{U} \varphi)(y) & =\int \frac{d p_{x}}{2 \pi \hbar} e^{i K\left(y, p_{x}\right)} \tilde{\varphi}\left(p_{x}\right), \\
e^{i K\left(y, p_{x}\right)} & =\int d x e^{i\left[F(y, x)+x p_{x} / \hbar\right]},
\end{aligned}
$$

alternatively, in the momentum-momentum representation we have

$$
\begin{aligned}
(\hat{U} \tilde{\varphi})\left(p_{y}\right) & =\int \frac{d p_{x}}{2 \pi \hbar} e^{i H\left(p_{y}, p_{x}\right)} \tilde{\varphi}\left(p_{x}\right), \\
e^{i H\left(p_{y}, p_{x}\right)} & =\int d q e^{-i q\left(p_{x}-p_{y}\right) / \hbar} u\left(\frac{p_{y}+p_{x}}{2}, q\right) .
\end{aligned}
$$

For the other mixed case

$$
\begin{aligned}
& (\hat{U} \tilde{\varphi})\left(p_{y}\right)=\int d x e^{i L\left(p_{y}, x\right)} \varphi(x), \\
& e^{i L\left(p_{y}, x\right)}=\int \frac{d p_{x}}{2 \pi \hbar} e^{i\left[H\left(p_{y}, p_{x}\right)-x p_{x} / \hbar\right]} .
\end{aligned}
$$

Hilbert space representations of canonical transformations like (24)-(27) have been written by Dirac using intuitive arguments in his celebrated book on quantum mechanics [2]. Here a direct proof of his results is presented using the Weyl correspondence.

Note that we have not assumed any particular property for the generic unitary operator $\hat{U}$. Now we assume that $\hat{U}$ produces the canonical transformation

$\hat{P}=\hat{U}^{-1} \hat{p} \hat{U}, \quad \hat{Q}=\hat{U}^{-1} \hat{q} \hat{U}$.

Multiplying both sides by $\hat{U}$ on the left and using the Weyl correspondence in Eq. (3) we find

$u(p, q) \star Q(p, q)=q \star u(p, q)=\left(q+\frac{i \hbar}{2} \frac{\partial}{\partial p}\right) u$,
$u(p, q) \star P(p, q)=p \star u(p, q)=\left(p-\frac{i \hbar}{2} \frac{\partial}{\partial q}\right) u$,

where $\star=\star q, p$ as defined in (4). Another crucial property of the $\star$-product is that, $\star=\star \star_{q, p}=\star Q, P$. This can be easily seen from (4) considering that $p, q$ and $P, Q$ are related by a CT. Once Eqs. (29b) are solved, the generators of the CT can be found by using Eqs. (24)-(27).

\section{Examples}

Let us solve the Eqs. (29a) and (29b) for a few well known cases. We first do it for the group of linear symplectic transformations $S L_{2}(\mathbb{R})$.

(a) $S L_{2}(\mathbb{R})$ :

In this case we have

$$
\left(\begin{array}{l}
P \\
Q
\end{array}\right)=g\left(\begin{array}{l}
p \\
q
\end{array}\right), \quad g=\left(\begin{array}{ll}
a & b \\
c & d
\end{array}\right) \in S L_{2}(\mathbb{R}) .
$$

Directly using (30) in (29a) and (29b) one has

$$
\begin{aligned}
u(p, q)= & \frac{2}{\sqrt{a+d+2}} \exp \left\{\frac{-2 i}{(a+d+2) \hbar}\right. \\
& \left.\times\left[b q^{2}+c p^{2}-(a-d) p q\right]\right\},
\end{aligned}
$$

where $\operatorname{Tr} g \neq-2$ and the normalization is chosen such that identity transformation is $u(p, q)=1$. By (24) this can be converted into the kernel

$e^{i F(y, x)}=\frac{e^{-i \pi / 4}}{\sqrt{2 \pi \hbar c}} e^{\frac{-i}{2 \hbar c}\left(a y^{2}+d x^{2}-2 x y\right)}$

yielding the correct integral kernel for $S L_{2}(\mathbb{R})$ transformation including the normalization factor [4]. The special cases such as $\operatorname{Tr} g=-2$ can be treated with additional limiting procedures which will not be considered here.

(b) Linear potential:

The second exactly solvable system is the linear potential model

$\left(\begin{array}{l}P \\ Q\end{array}\right)=\left(\begin{array}{c}p \\ q+a p^{2}\end{array}\right), \quad a \in \mathbb{R}$

using (29a) and (29b) once more we find,

$u(p, q)=N_{a} \exp \left(-\frac{i a}{3 \hbar} p^{3}\right),\left.\quad N_{a}\right|_{a=0}=1$

which is more conveniently used in a mixed type of transformation kernel given by Eq. (25) as

$e^{i K\left(y, p_{x}\right)}=e^{\frac{-i}{h}\left(y p_{x}-\frac{a}{3} p_{x}^{3}\right)}$,

where $N_{a}=1$ is used, yielding the correct solution of the linear potential model [18]. Also unphysical $\hbar$ dependencies may appear if the Moyal covariance is not correctly taken into account [6].

In both examples the unitary transformation kernel $u(p, q)$ is closely related to the appropriate classical generating function of the canonical transform as remarked by Dirac [2] in the early days of the quantum theory. A close look into (32) as well as (35) confirms that they are exponentiated versions of one of the four types of generating functions that one learns in the textbooks. An important remark is that, since the quantum and classical generating functions are identical, there are no $\hbar$-corrections as anticipated in some earlier works [6]. Indeed, (32) is, after renaming $y \rightarrow Q$ and $x \rightarrow q$ as the new and 
the old coordinates

$F_{1}^{(\mathrm{q})}(Q, q)=-\frac{1}{2 c}\left(a Q^{2}+d q^{2}-2 Q q\right)$

which is just the classical generating function $F_{1}^{(\mathrm{cl})}(Q, q)$ for the linear symplectic transformations satisfying $p=\frac{\partial F_{1}^{(\mathrm{cl})}(Q, q)}{\partial q}$ and $P=-\frac{\partial F_{1}^{(\mathrm{cl})}(Q, q)}{\partial Q}$.

Likewise, in Eq. (35) the quantum generator (in the notation $y \rightarrow Q$ and $p_{x} \rightarrow p$ ) is

$F_{3}^{(\mathrm{q})}(Q, p)=-Q p+\frac{a}{3} p^{3}$

which is just the classical generating function $F_{3}^{(\mathrm{cl})}(Q, p)$ for the non-linear transformation in Eq. (33) satisfying $q=$ $-\partial F_{3}^{(\mathrm{cl})}(Q, p) / \partial p$ and $P=-\partial F_{3}^{(\mathrm{cl})}(Q, p) / \partial Q$. Eq. (35) that was found for the linear potential model matches exactly with the exponentiated classical generator and agrees with Dirac's exponentiation formula [2].

Eq. (7) provides some background we need in order to understand the solutions of (29a) and (29b) for the class of problems for which $u(p, q)$ has no $\hbar$-corrections. The $\hbar$-corrections to the CT generators were proposed in Ref. [15] in reference to a particular Hamiltonian. This concept can be made independent of a dynamical model by demanding that the solution of (29a) and (29b) yields integral kernels $F_{1}(Q, q), F_{2}(q, P), F_{3}(Q, p), F_{4}(P, p)$ in (24)-(27) which are all in the order of $1 / \hbar$ independent from any class of Hamiltonians considered implied by

$u(p, q)=e^{\frac{2 i}{\hbar} T(p, q)}, \quad \frac{\partial T}{\partial \hbar}=0$

hence $T(p, q)$ has no $\hbar$ dependence and the corresponding generating functions $F_{1}, F_{2}, F_{3}, F_{4}$ in (24)-(27) are identical to their classical counterparts.

By inspecting Eqs. (29a) and (29b) one expects to find that the particular class of transformations for which

$$
\begin{aligned}
& u(p, q) \star_{q, p} Q(p, q)=u(p, q) \star_{Q, P} Q, \\
& u(p, q) \star_{q, p} P(p, q)=u(p, q) \star_{Q, P} P
\end{aligned}
$$

holds, yields $\hbar$-uncorrected solutions as in Eq. (38) for $u(p, q)$. It is intuitive that the conditions in (39) and (40) are sufficient but not necessary for the $\hbar$-uncorrected solutions in (38). If Eqs. (39) and (40) hold, then

$$
\begin{aligned}
& \left(Q-\frac{i \hbar}{2} \partial_{P}\right) u(p, q)=\left(q+\frac{i \hbar}{2} \partial_{p}\right) u(p, q), \\
& \left(P+\frac{i \hbar}{2} \partial_{Q}\right) u(p, q)=\left(p-\frac{i \hbar}{2} \partial_{q}\right) u(p, q) .
\end{aligned}
$$

Considering the general form in (38) the solution is

$$
\begin{aligned}
\left(\begin{array}{c}
\partial_{p} \\
\partial_{q}
\end{array}\right) T= & \left(2+\partial_{P} p+\partial_{Q q}\right)^{-1} \\
& \times\left(\begin{array}{cc}
1+\partial_{Q} q & -\partial_{P} q \\
-\partial_{Q} p & 1+\partial_{P} p
\end{array}\right)\left(\begin{array}{c}
q-Q \\
P-p
\end{array}\right)
\end{aligned}
$$

here it is required that the determinant of the matrix $(2+$ $\left.\partial_{P} p+\partial_{Q} q\right)$ is non-zero and we employed the Lagrange bracket $\{q, p\}_{Q, P}=1$ as a canonical invariant. The solution to (43) is clearly $\hbar$ independent if the canonical transformation $(p, q) \mapsto$ $(P, Q)$ is also independent of $\hbar$. Eqs. (41) and (42) are manifestly satisfied for the linear symplectic transformations in Eq. (30). Our interest was based on the validity of (7), but a broader class of CTs via the Weyl-Wigner formalism as in [20] will be subject of another work.

\section{Conclusions}

In this work we introduced Weyl's phase space representations of the non-linear quantum canonical transformations. We have shown that the non-linear canonical transformations which generally lack unitary representations in Hilbert space, have unitary phase space representations.

It has been believed for a long time that Weyl quantization did not possess covariance under non-linear CT. As the results in this work indicate, different Weyl representations can be connected by the non-linear CT thereby extending the concept of covariance instead of breaking it. Another advantage in seeing this as an extended covariance is that the presented approach also unifies with Dirac's transformation theory which is essentially a Hilbert space approach. Dirac's transformation theory can be naturally merged [as shown in Section 4] with Weyl's phase space approach bringing the theory of CT (particularly non-linear, invertible) back to where it should belong.

Nearly as old as the quantum mechanics itself, the Weyl quantization remains to be one of the most active fields in a wide area of physics. Without need of mentioning its applications in quantum and classical optics, condensed matter physics and engineering [21], it has been put into a more general frame in the deformation quantization [17]. Recently, it also proved to be an essential part of the non-commutative quantum field and string theories in the presence of background gauge fields [22]. It is then natural to expect that the theory of canonical transformations, which is subject to progress within itself, may also find some applications in these new directions.

\section{Acknowledgements}

The author T.H. is thankful to C. Zachos (High Energy Physics Division, Argonne National Laboratory) for stimulating discussions. This work was supported in part by TÜBİTAK (Scientific and Technical Research Council of Turkey), Bilkent University and the US Department of Energy, Division of High Energy Physics, under contract W-31-109-Eng-38.

\section{References}

[1] M. Born, W. Heisenberg, P. Jordan, Z. Phys. 35 (1926) 557; P. Jordan, Z. Phys. 57 (1926) 383; P. Jordan, Z. Phys. 38 (1926) 513

[2] P.A.M. Dirac, Physik Z. Sowjetunion 3 (1933) 64; P.A.M. Dirac, The Principles of Quantum Mechanics, Oxford Univ. Press, Oxford, 1958 
[3] M. Jammer, The Conceptual Development of Quantum Mechanics, McGraw-Hill, New York, 1966, Ch. 6.2.

[4] K.B. Wolf, Integral Transforms in Science and Engineering, Plenum, New York, 1979.

[5] B. Leaf, J. Math. Phys. 10 (1969) 1971;

B. Leaf, J. Math. Phys. 10 (1969) 1980;

P.A. Mello, M. Moshinsky, J. Math. Phys. 16 (1975) 2017;

P. Kramer, M. Moshinsky, T. Seligman, J. Math. Phys. 19 (1978) 683;

M. Moshinsky, T. Seligman, Ann. Phys. 114 (1978) 243;

J. Phys. A 12 (1979) L135.

[6] A. Dragt, S. Habib, How Wigner functions transform under symplectic maps, in: Proceedings of the Advanced Beam Dynamics Workshop on Quantum Aspects of Beam Physics, quant-ph/9806056.

[7] A. Anderson, Ann. Phys. 232 (1994) 292, hep-th/9305054.

[8] A.O. Barut, I.H. Duru, Phys. Rev. A 38 (1988) 5906.

[9] J. Hietarinta, Phys. Rev. D 25 (1982) 2103.

[10] V. Guillemin, S. Sternberg, Symplectic Techniques in Physics, Cambridge Univ. Press, Cambridge, 1984.

[11] H. Weyl, Z. Phys. 46 (1927) 1.

[12] J. von Neumann, Math. Ann. 104 (1931) 570; E.P. Wigner, Phys. Rev. 40 (1932) 749;

H.J. Groenewold, Physica 12 (1946) 405;
J.E. Moyal, Proc. Cambridge Philos. Soc. 45 (1949) 99.

[13] C. Zachos, T. Curtright, Prog. Theor. Phys. Suppl. 135 (1999) 244.

[14] T. Curtright, D. Fairlie, C. Zachos, Phys. Rev. D 58 (1998) 025002.

[15] T. Hakioğlu, A.J. Dragt, J. Phys. A 34 (2001) 6603.

[16] G.I. Ghandour, Phys. Rev. D 35 (1987) 1289;

E.D. Davis, G.I. Ghandour, quant-ph/9905002.

[17] M. Flato, A. Lichnerowicz, D. Sternheimer, J. Math. Phys. 17 (1975) 1754;

F. Bayen, M. Flato, C. Fronsdal, A. Lichnerowicz, D. Sternheimer, Ann. Phys. 110 (1978) 111;

F. Bayen, M. Flato, C. Fronsdal, A. Lichnerowicz, D. Sternheimer, Ann. Phys. 111 (1978) 61.

[18] E. Braaten, T. Curtright, C. Thorn, Ann. Phys. 147 (1983) 365.

[19] A. Verçin, Ann. Phys. 266 (1998) 503;

T. Dereli, A. Verçin, J. Math. Phys. 38 (1997) 5515.

[20] A.J. Bracken, G. Cassinelli, J.G. Wood, J. Phys. A 36 (2003) 1033.

[21] G.W. Forbes, V. Man'ko, H. Ozaktas, R. Simon, K.B. Wolf, J. Opt. Soc. Am. A 17 (2000) 2274.

[22] A. Connes, Noncommutative Geometry, Academic Press, San Diego, 1994;

R. Gopakumar, S. Minvalla, A. Strominger, hep-th/0003160; N. Seiberg, E. Witten, JHEP 9909 (1999) 032. 